\title{
Subset of the Shape of Numbers
}

\section{Ji Peng}

Department of Electronic Information, Nanjing University, Nanjing, China

Email: mcfroo@sina.com

How to cite this paper: Peng, J. (2020) Subset of the Shape of Numbers. Open Access Library Journal, 7: e7040.

https://doi.org/10.4236/oalib.1107040

Received: November 26, 2020

Accepted: December 28, 2020

Published: December 31, 2020

Copyright $\odot 2020$ by author(s) and Open Access Library Inc.

This work is licensed under the Creative Commons Attribution International License (CC BY 4.0).

http://creativecommons.org/licenses/by/4.0/

\begin{abstract}
This article is based on the concept of Shape of numbers, introduces subset of the Shape and obtains its calculation formula. This article also makes some analysis and draws new conclusions, especially the calculation method of $1^{M}+2^{M}+3^{M}+\cdots+N^{M}$. The Shape's concept becomes clearer and richness.
\end{abstract}

\section{Subject Areas}

Discrete Mathematics

\section{Keywords}

Shape of Numbers, Calculation Formula, Sum of Powers of Integers, Combinatorics, Congruence, Stirling Number

\section{Introduction}

Peng, J. has introduced Shape of numbers in [1] [2]:

$$
\left(I_{1}, I_{2}, \cdots, I_{M}\right), I_{i} \in N, I_{1}<I_{2}<\cdots<I_{M}
$$

There are $M$ - 1 intervals between adjacent numbers.

$I_{i+1}-I_{i}=1$ means continuity, $I_{i+1}-I_{i}>1$ means discontinuity.

Shape of numbers: collect $\left(I_{1}, I_{2}, \cdots, I_{M}\right)$ with the same continuity and discontinuity at the same position into a catalog, call it a Shape. A Shape has a min Item:

$\left(1, K_{1}, K_{2}, \cdots\right)$, Use the symbol $P S=$ [min Item] to represent it. If $K_{i+1}-K_{i}=D>1$ then only $I_{i+1}-I_{i} \geq D$ is allowed.

The single $\left(I_{1}, I_{2}, \cdots, I_{M}\right)$ is an item, $I_{1} I_{2} \cdots I_{M}$ is the product. $I_{i}$ is a factor.

Example:

$$
P S=[1,2] \rightarrow(1,2),(2,3),(3,4),(1000,1001) \in P S
$$




$$
\begin{aligned}
& P S=[1,3] \rightarrow(1,3),(1,4),(2,4),(1,5),(2,5),(3,5),(1000,2001) \in P S \\
& P S=[1,4] \rightarrow(1,4),(1,5),(2,5),(1,6),(2,6),(3,6) \in P S,(3,5),(4,6) \notin P S \\
& P S=[1,4,6] \rightarrow(1,4,7),(1,5,7),(2,5,7) \in P S,(3,5,7) \notin P S
\end{aligned}
$$

Define:

$S E T(N, P S)=$ set of items belonging to PS in $[1, N-1]$

$P M(P S)=$ count of factors

$P B(P S)=$ count of discontinuities

$M I N(P S)=$ min product: $\operatorname{MIN}([1,2,3])=1 \times 2 \times 3, \quad M I N([1,2,4])=1 \times 2 \times 4$

$I D X(P S)=(\max$ factor $)+1$

$P H(P S)=I D X(P S)-P B(P S)-2$

Basic Shape: intervals $=1$ or 2

$B A S E(P S)=B S:$ if 1) $P B(B S)=P B(P S)$, 2) $P M(B S)=P M(P S), 3) B S$ is a

Basic Shape 4) $B S$ has discontinuity intervals at the same positions of $P S$.

Example:

$$
\begin{aligned}
& P S=[1,2] \rightarrow B A S E(P S)=[1,2] \\
& P S=[1,3],[1,4],[1, K>2] \rightarrow B A S E(P S)=[1,3] \\
& P S=[1,3,4],[1,4,5],[1, K>2, X=K+1] \rightarrow B A S E(P S)=[1,3,4] \\
& P S=[1,3,5],[1,4,9],[1, K>2, X>K+1] \rightarrow B A S E(P S)=[1,3,5] \\
& |\operatorname{SET}(\boldsymbol{N}, P S)|=\text { Count of items in } S E T(N, P S) \\
& \operatorname{SUM}(\boldsymbol{N}, P S)=\text { Sum of all products in } S E T(N, P S)
\end{aligned}
$$

Example:

$$
\begin{aligned}
& \operatorname{SUM}(6,[1,2,4])=1 \times 2 \times 4+1 \times 2 \times 5+2 \times 3 \times 5 \\
& \operatorname{SUM}(9,[1,4,7])=1 \times 4 \times 7+1 \times 4 \times 8+1 \times 5 \times 8+2 \times 5 \times 8
\end{aligned}
$$

[1] [2] came to the following conclusion:

1.1) $|\operatorname{SET}(N, P S)|=\left(\begin{array}{c}N-P H(P S)-1 \\ P B(P S)+1\end{array}\right)$

1.2) $\operatorname{SUM}(N, P S)=\operatorname{MIN}(P S)\left(\begin{array}{c}N \\ I D X(P S)\end{array}\right), P S$ is a Basic Shape

The following uses count of $X \in K$ for count of $\left\{X_{1}, X_{2}, \cdots, X_{M}\right\} \in\left\{K_{1}, K_{2}, \cdots, K_{M}\right\}$

1.3) $P S=\left[1, K_{1}, \cdots, K_{M}\right], B S=B A S E(P S)=\left[1, G_{1}, \cdots, G_{M}\right]$

Use the form $\left(G_{1}+K_{1}\right)\left(G_{2}+K_{2}\right) \cdots\left(G_{M}+K_{M}\right)=\sum X_{1} X_{2} \cdots X_{M}, X_{i}=G_{i}$ or $K_{r}$

The expansion has $2^{M}$ items, don't swap the factors of $X_{1} X_{2} \cdots X_{M}$, then each $X_{1} X_{2} \cdots X_{M}$ corresponds to one expression $=A_{q}\left(\begin{array}{l}N-P H(P S) \\ I D X(B S)-q\end{array}\right), \boldsymbol{q}=$ count of $X \in K$

$$
S U M(N, P S)=\sum A_{q}\left(\begin{array}{l}
N-P H(P S) \\
I D X(B S)-q
\end{array}\right), 2^{M} \text { items in total. }
$$


$A_{q}=\prod_{i=1}^{M}\left(X_{i}+D_{i}\right), \quad D_{i}=\left\{\begin{array}{l}-m: X_{i}=G_{i}, m=\text { count of }\left\{X_{1}, \cdots, X_{i-1}\right\} \in K \\ +m: X_{i}=K_{i}, m=\text { count of }\left\{X_{1}, \cdots, X_{i-1}\right\} \in G\end{array}\right.$

Example:

$P S=\left[1, K_{1} \geq 3, K_{2} \geq K_{1}+2, K_{3} \geq K_{2}+2\right], \quad B S=\operatorname{BASE}(P S)=[1,3,5,7]$

The form $=\left(3+K_{1}\right)\left(5+K_{2}\right)\left(7+K_{3}\right)$

$$
\begin{aligned}
= & 3 \times 5 \times 7+3 \times 5 \times K_{3}+3 \times K_{2} \times 7+3 \times K_{2} \times K_{3} \\
& +K_{1} \times 5 \times 7+K_{1} \times 5 \times K_{3}+K_{1} \times K_{2} \times 7+K_{1} \times K_{2} \times K_{3}
\end{aligned}
$$

$P=N-P H(P S)=N-\{I D X(P S)-P B(P S)-2\}$

$=N-\left\{K_{3}+1-3-2\right\}=N-K_{3}+4$

$\operatorname{IDX}(B S)=8$

$$
\begin{aligned}
\operatorname{SUM}(N, P S)= & 3 \times 5 \times 7\left(\begin{array}{l}
P \\
8
\end{array}\right)+3 \times 5 \times\left(K_{3}+2\right)\left(\begin{array}{l}
P \\
7
\end{array}\right) \\
& +3 \times\left(K_{2}+1\right) \times(7-1)\left(\begin{array}{l}
P \\
7
\end{array}\right)+3 \times\left(K_{2}+1\right) \times\left(K_{3}+1\right)\left(\begin{array}{l}
P \\
6
\end{array}\right) \\
& +K_{1} \times(5-1) \times(7-1)\left(\begin{array}{l}
P \\
7
\end{array}\right)+K_{1} \times(5-1) \times\left(K_{3}+1\right)\left(\begin{array}{l}
P \\
6
\end{array}\right) \\
& +K_{1} \times K_{2} \times(7-2)\left(\begin{array}{l}
P \\
6
\end{array}\right)+K_{1} \times K_{2} \times K_{3}\left(\begin{array}{l}
P \\
5
\end{array}\right)
\end{aligned}
$$

An item $\in P S=\left\{\right.$ begin, $\left.K_{1}+E_{1}, \cdots, K_{M}+E_{M}\right\}, K$ is fixed, $E$ is variable.

A product $=$ begin $\times\left(K_{1}+E_{1}\right) \cdots\left(K_{M}+E_{M}\right)=\operatorname{begin} \times \sum F_{1} F_{2} \cdots F_{M} \quad, \quad F_{i}=E_{i}$ or $F_{i}=K_{i}$

That is, a product can be broken down into $2^{M}$ parts.

\section{Define}

$S U M_{-} K\left(\operatorname{SET}(N, P S), P F=F_{1} F_{2} \cdots F_{M}\right)=$ Sum of one part of $\operatorname{SUM}(N, P S)$

$P F$ indicates the part. $P F=F_{1} F_{2} \cdots F_{M}, F_{i}=E_{i}$ or $F_{i}=K_{i}$

Rewrite 1.3) and add \{braces\}:

$$
\begin{gathered}
\operatorname{SUM}(N, P S)=\sum \text { product }=\sum \sum \text { begin } \times F_{1} \cdots F_{M} \\
=\sum \prod_{i=1}^{M}\left(X_{i}+D_{i}\right)\left(\begin{array}{c}
A \\
M_{q}
\end{array}\right) \\
X_{i}+D_{i}=\left\{\begin{array}{l}
\left\{G_{i}-D_{i}\right\}: X_{i}=G_{i}, D_{i}=\text { count of }\left\{X_{1}, \cdots, X_{i-1}\right\} \in K \\
\left\{K_{i}\right\}+\left\{D_{i}\right\}: X_{i}=K_{i}, D_{i}=\text { count of }\left\{X_{1}, \cdots, X_{i-1}\right\} \in G
\end{array}\right.
\end{gathered}
$$

Let $\operatorname{SUM} 1(N, P S)=S U M(N, P S)$ expand by the $\{$ braces $\}$ :

1.4) $S U M \_K(S E T(N, P S), P F)=\sum$ Expansion of $S U M 1(N, P S)$ with same

$$
\left\{K_{i}\right\} \in P F=\sum \prod_{i=1}^{M} Y_{i}\left(\begin{array}{c}
A \\
M_{q}
\end{array}\right),
$$

$$
Y_{i}=\left\{\begin{array}{l}
0: F_{i}=K_{i}, X_{i}=G_{i} \\
K_{i}: F_{i}=K_{i}, X_{i}=K_{i} \\
G_{i}-D_{i}: F_{i}=E_{i}, X_{i}=G_{i}, D_{i}=\operatorname{count} \text { of }\left\{X_{1}, \cdots, X_{i-1}\right\} \in K \\
D_{i}: F_{i}=E_{i}, X_{i}=K_{i}, D_{i}=\text { count of }\left\{X_{1}, \cdots, X_{i-1}\right\} \in G
\end{array}\right.
$$


Example:

$$
\begin{aligned}
& \operatorname{SUM}\left(N,\left[1, K_{1} \geq 3, K_{2} \geq K_{1}+2\right]\right), \text { form }=\left(3+K_{1}\right)\left(5+K_{2}\right) \rightarrow \\
& =15\left(\begin{array}{c}
N-K_{2}+3 \\
6
\end{array}\right)+3\left(\left\{K_{2}\right\}+\{1\}\right)\left(\begin{array}{c}
N-K_{2}+3 \\
5
\end{array}\right) \\
& \quad+K_{1}(\{5-1\})\left(\begin{array}{c}
N-K_{2}+3 \\
5
\end{array}\right)+K_{1} K_{2}\left(\begin{array}{c}
N-K_{2}+3 \\
4
\end{array}\right)
\end{aligned}
$$

Expand by the $\{$ braces $\}$ :

$$
\begin{aligned}
& =\left\{15\left(\begin{array}{c}
N-K_{2}+3 \\
6
\end{array}\right)+3\left(\begin{array}{c}
N-K_{2}+3 \\
5
\end{array}\right)\right\}+3 K_{2}\left(\begin{array}{c}
N-K_{2}+3 \\
5
\end{array}\right) \\
& +4 K_{1}\left(\begin{array}{c}
N-K_{2}+3 \\
5
\end{array}\right)+K_{1} K_{2}\left(\begin{array}{c}
N-K_{2}+3 \\
4
\end{array}\right) \\
& =\sum_{\text {begin }=1}^{N-K_{2}} \sum \text { begin } \times\left(K_{1}+E_{1, \text { begin }}\right)\left(K_{2}+E_{2, \text { begin }}\right) \\
& \rightarrow \\
& S U M_{-} K\left(S E T(N, P S), E_{1} E_{2}\right) \\
& =\sum_{\text {all items }} \text { begin } * E_{1, i} E_{2, i}=15\left(\begin{array}{c}
N-K_{2}+3 \\
6
\end{array}\right)+3\left(\begin{array}{c}
N-K_{2}+3 \\
5
\end{array}\right) \\
& S U M_{-} K\left(S E T(N, P S), E_{1} K_{2}\right)=\sum_{\text {all items }} \text { begin } * E_{1, i} K_{2}=3 K_{2}\left(\begin{array}{c}
N-K_{2}+3 \\
5
\end{array}\right) \\
& S U M_{-} K\left(S E T(N, P S), K_{1} E_{2}\right)=\sum_{\text {all items }} \text { begin } * K_{1} E_{2, i}=4 K_{1}\left(\begin{array}{c}
N-K_{2}+3 \\
5
\end{array}\right) \\
& S U M_{-} K\left(S E T(N, P S), K_{1} K_{2}\right)=\sum_{\text {all items }} \text { begin } * K_{1} K_{2}=K_{1} K_{2}\left(\begin{array}{c}
N-K_{2}+3 \\
4
\end{array}\right)
\end{aligned}
$$

This can explain why 1.3) has that strange form:

We can calculate every part of 1.3 ) by some way without 1.3 ). There may be complex relationships between the parts, but their sum just match a simple form.

1.5) Use the symbol of 1.3), when $G_{i}=K_{i}, \quad N_{1}=$ Count of $X \in K$, $N_{1}+N_{2}=M$

$$
H\left(N_{1}, N_{2}, K\right)=\sum A_{q=N_{1}}=\sum \prod_{i=1}^{M}\left(X_{i}+D_{i}\right)=K_{1} K_{2} \cdots K_{M}\left(\begin{array}{c}
M \\
N_{1}, N_{2}
\end{array}\right)
$$

\section{Sum traverses all $\left(N_{1}, N_{2}\right)$-Choice of $K$}

This $\rightarrow 1.3$ ) is compatible with 1.2)

1.6) $P$ is a prime number, $\{P S 1, P S 2, \ldots\}$ are all of the Basic Shapes, $P M(P S 1)=P M(P S 2)=\cdots, P B(P S 1)=P B(P S 2)=\cdots>0$, $I D X(P S 1)=I D X(P S 2)=\cdots=P$,

That is, them are Basic shapes, have same count of factors and same count of discontinuities $>0$, and $\max$ factor $=P-1$, then $\operatorname{MIN}(P S 1)+\operatorname{MIN}(P S 2)+\cdots \equiv 0 M O D P$

Example:

$$
\begin{aligned}
& 1 \times 2 \times 4 \times 6+1 \times 3 \times 4 \times 6+1 \times 3 \times 5 \times 6 \\
& \equiv 1 \times 2 \times 3 \times 4 \times 6+1 \times 2 \times 3 \times 5 \times 6+1 \times 2 \times 4 \times 5 \times 6+1 \times 3 \times 4 \times 5 \times 6 \\
& \equiv 0 M O D 7
\end{aligned}
$$




\section{Subset of $\operatorname{SET}(N, P S)$}

$$
\begin{aligned}
& P S=\left[1, K_{1}, K_{2}, \cdots, K_{M}\right], \quad P T=\left[1, T_{1}, T_{2}, \cdots, T_{M}\right], \\
& \text { Item }=\left\{I_{0}, I_{1}, I_{2}, \cdots, I_{M}\right\} \in \operatorname{SET}(N, P S) \\
& \text { If } P B(P S)=0 \text {, items } \in S E T(N, P S) \text { is very simple. } \\
& \text { If } P B(P S)>0 \text {, some changes appear in } S E T(N, P S) \text {. } \\
& \text { We can fix some discontinuities of the Shape to get subsets. } \\
& P T=\left[1, T_{1}, \cdots, T_{M}\right] \\
& =\left\{\begin{array}{l}
T_{i+1}-T_{i}=1: K_{i+1}-K_{i}=1, \text { means } I_{i+1}-I_{i}=1 \\
T_{i+1}-T_{i}=1: K_{i+1}-K_{i}=D>1, \text { means } I_{i+1}-I_{i}=D \\
T_{i+1}-T_{i}=2: K_{i+1}-K_{i}=D>1, \text { means } I_{i+1}-I_{i} \geq D
\end{array}\right.
\end{aligned}
$$

others are invalid.

Example:

$\operatorname{SET}(N,[1,3,5],[1,3,5])=\operatorname{SET}(N,[1,3,5])$

$\operatorname{SET}(N,[1,3,5],[1,4,5]), \quad \operatorname{SET}(N,[1,3,5],[1,3,6]), \quad \operatorname{SET}(N,[1,2,9],[1,3,4])$ is invalid.

$$
\begin{aligned}
& \operatorname{SET}(N,[1,3,5],[1,2,4]) \\
& =\{(1,3,5),(1,3,6),(2,4,6),(1,3,7),(2,4,7),(3,5,7), \cdots\} \\
& I_{2}-I_{1}=(3-1)=2, I_{3}-I_{2} \geq(5-3)=2 \\
& \operatorname{SET}(N,[1,3,5],[1,3,4]) \\
& =\{(1,3,5),(1,4,6),(2,4,6),(1,5,7),(2,5,7),(3,5,7), \cdots\} \\
& I_{3}-I_{2}=(5-3)=2, I_{2}-I_{1} \geq(3-1)=2 \\
& \operatorname{SET}(N,[1,3,5],[1,2,3])=\{(1,3,5),(2,4,6),(3,5,7), \cdots\} \\
& \operatorname{SET}(N,[1,4,8],[1,2,4]) \\
& =\{(1,4,8),(1,4,9),(2,5,9),(1,4,10),(2,5,10),(3,6,10), \cdots\} \\
& I_{2}-I_{1}=(4-1)=3, I_{3}-I_{2} \geq(8-4)=4
\end{aligned}
$$

PT only has the change at $\left.{ }^{*}\right)$. When a change happens, make the interval fixed.

The more changes, the fewer items:

\section{Define $P C H G(P S, P T)=$ count of change from $B A S E(P S)$ to $P T$}

Example:

$\operatorname{PCHG}([1,3,5],[1,2,4])=\operatorname{PCHG}([1,4,7],[1,2,4])=1$, changed at $T_{1}$

$\operatorname{PCHG}([1,3,5],[1,3,4])=\operatorname{PCHG}([1,4,7],[1,3,4])=1$, changed at $T_{2}$

$\operatorname{PCHG}([1,3,5],[1,2,3])=\operatorname{PCHG}([1,8,10],[1,2,3])=2$, changed at $T_{1}, T_{2}$

2.1) $\operatorname{SET}(N, P S)=\operatorname{SET}(N, P S, B A S E(P S))$

2.2) $|\operatorname{SET}(N, P S, P T)|=\left(\begin{array}{c}N-P H(P S)-1-P C H G(P S, P T) \\ P B(P T)+1\end{array}\right)$ 
If PT1 only change $T_{i}$ of $P T$, Obvious: $P C H G(P S, P T 1)=P C H G(P S, P T)+1$

2.3) If $P T 1$ only change $T_{i}$ of $P T$,

$P T 1=\left[1, T_{1}, \cdots, T_{i-1}, T_{i}-1, T_{i+1}-1, \cdots, T_{M}-1\right]$.

Let $P S 1=\left[1, K_{1}, \cdots, K_{i-1}, K_{i}+1, K_{i+1}+1, \cdots, K_{M}+1\right]$, then

$\operatorname{SET}(N, P S, P T)=\operatorname{SET}(N, P S, P T 1) \cup \operatorname{SET}(N, P S 1, P T)$

$\operatorname{SET}(N, P S, P T 1)=\operatorname{SET}(N, P S, P T)-\operatorname{SET}(N, P S 1, P T)$

In particular: $P C H G(P S, P T)=1 \rightarrow$

$\operatorname{SET}(N, P S, P T)=\operatorname{SET}(N, P S)-\operatorname{SET}(N, P S 1)$

[Proof]

PT1 change $T_{i}$ of $P T \rightarrow T_{i}-T_{i-1}=2 \rightarrow K_{i}-K_{i-1}>1 \rightarrow$ $P C H G(P S, P T)=P C H G(P S 1, P T)$

$$
\begin{aligned}
\mid & \operatorname{SET}(N, P S, P T 1)|+| S E T(N, P S 1, P T) \mid \\
= & \left(\begin{array}{c}
N-P H(P S)-1-P C H G(P S, P T 1) \\
P B(P T 1)+1
\end{array}\right) \\
& +\left(\begin{array}{c}
N-P H(P S 1)-1-P C H G(P S 1, P T) \\
P B(P T)+1
\end{array}\right) \\
& +\left(\begin{array}{c}
N-P H(P S)-2-P C H G(P S, P T) \\
P B(P T)
\end{array}\right) \\
= & \left(\begin{array}{c}
N-P H(P S)-2-P C H G(P S, P T) \\
P B(P T)
\end{array}\right) \\
& +\left(\begin{array}{c}
N-P H(P S)-2-P C H G(P S, P T) \\
P B(P T)+1
\end{array}\right) \\
= & \left(\begin{array}{c}
N-P H(P S)-1-P C H G(P S, P T) \\
P B(P T)+1
\end{array}\right) \\
= & |S E T(N, P S, P T)|
\end{aligned}
$$

Count of the Items is equal.

Every item in $S E T(N, P S 1, P T)$ is in $\operatorname{SET}(N, P S, P T)$, and not in $S E T(N, P S$, PT1).

q.e.d.

if $P C H G(P S, P T)=2, P T$ changes at $T_{i}$ and $T_{p}$ then

$$
P T=\left[1, G_{1}, \cdots, G_{i-1}, G_{i}-1, \cdots, G_{j-1}-1, G_{j}-2, G_{j+1}-2, \cdots, G_{M}-2\right]
$$

Let

$$
\begin{aligned}
& P T A=\left[1, G_{1}, \cdots, G_{i-1}, G_{i}-1, \cdots, G_{M}-1\right], \\
& P T B=\left[1, G_{1}, \cdots, G_{j-1}, G_{j}-1, \cdots, G_{M}-1\right] \\
& P S A=\left[1, K_{1}, \cdots, K_{i-1}, K_{i}+1, \cdots, K_{M}+1\right],
\end{aligned}
$$




$$
\begin{aligned}
& P S B=\left[1, K_{1}, \cdots, K_{j-1}, K_{j}+1, \cdots, K_{M}+1\right] \\
& P S 2=\left[1, K_{1}, \cdots, K_{i-1}, K_{i}+1, \cdots, K_{j-1}+1, K_{j}+2, K_{j+1}+2, \cdots, K_{M}+2\right] \\
& \rightarrow \\
& \operatorname{SET}(N, P S, P T)=\operatorname{SET}(N, P S, P T A)-\operatorname{SET}(N, P S A, P T A) \\
& =\{\operatorname{SET}(N, P S, B S)-S E T(N, P S B, B S)\} \\
& -\{\operatorname{SET}(N, P S A, B S)-\operatorname{SET}(N, P S 2, B S)\} \\
& =\operatorname{SET}(N, P S, B S)-[\operatorname{SET}(N, P S A, B S) \cup S E T(N, P S B, B S)] \\
& +\operatorname{SET}(N, P S 2, B S) \\
& =\operatorname{SET}(N, P S)-[\operatorname{SET}(N, P S A) \cup S E T(N, P S B)]+\operatorname{SET}(N, P S 2)
\end{aligned}
$$

General:

2.4) The relationship between $S E T(N, P S, P T)$ and $S E T(N, P S X)$ is similar to the Inclusion Exclusion Principle.

\section{Calculation formula of $\operatorname{SET}(N, P S, P T)$}

\section{Define:}

$S U M \_S U B S E T(N, P S, P T)=$ Sum of all products in $S E T(N, P S, P T)$ When $P T$ is invalid, $S U M \_S U B S E T(N, P S, P T)=0$

Only valid $P T$ is discussed below.

$$
\begin{gathered}
P S=\left[1, K_{1}, K_{2}, \cdots, K_{M}\right], \quad B S=B A S E(P S)=\left[1, G_{1}, G_{2}, \cdots, G_{M}\right], \\
P T=\left[1, T_{1}, T_{2}, \cdots, T_{M}\right]
\end{gathered}
$$

3.1) Use the form $\left(T_{1}+K_{1}\right)\left(T_{2}+K_{2}\right) \cdots\left(T_{M}+K_{M}\right)=\sum X_{1} X_{2} \cdots X_{M}$, then

$$
\begin{aligned}
& \text { SUM_SUBSET }(N, P S, P T)=\sum A_{q}\left(\begin{array}{c}
N-P H(P S)-P C H G(P S, P T) \\
I D X(P T)-q
\end{array}\right) \\
& A_{q}=\prod_{i=1}^{M}\left(X_{i}+D_{i}\right), D_{i}=\left\{\begin{array}{l}
-m: X_{i}=T_{i}, m=\operatorname{count} \text { of }\left\{X_{1}, \cdots, X_{i-1}\right\} \in K \\
+m: X_{i}=K_{i}, m=\operatorname{count} \text { of }\left\{X_{1}, \cdots, X_{i-1}\right\} \in T
\end{array}\right. \\
& q=\text { count of } X \in K
\end{aligned}
$$

[Proof]

1) If $P T=B S$, then $S U M_{-} S U B S E T(N, P S, B S)=S U M(N, P S) \rightarrow$ the formula holds.

2) If $M=1$ and $P T$ has 1 change, then $P S=\left[1, K_{1}>2\right], P T=\left[1, T_{1}\right]=[1,2]$, $B S=\left[1, G_{1}\right]=[1,3]$,

Let $\left.P S 1=\left[1, K_{1}+1\right], 2.3\right) \rightarrow$

$$
\begin{aligned}
& S U M_{-} S U B S E T(N, P S, P T) \\
= & S U M(N, P S)-S U M(N, P S 1) \\
= & \left\{G_{1}\left(\begin{array}{c}
N-P H(P S) \\
I D X(B S)
\end{array}\right)+K_{1}\left(\begin{array}{c}
N-P H(P S) \\
I D X(B S)-1
\end{array}\right)\right\} \\
& -\left\{G_{1}\left(\begin{array}{c}
N-P H(P S 1) \\
I D X(B S)
\end{array}\right)+\left(K_{1}+1\right)\left(\begin{array}{c}
N-P H(P S 1) \\
I D X(B S)-1
\end{array}\right)\right\}
\end{aligned}
$$




$$
\begin{aligned}
= & \left\{G_{1}\left(\begin{array}{c}
N-P H(P S 1) \\
I D X(B S)
\end{array}\right)+K_{1}\left(\begin{array}{c}
N-P H(P S 1) \\
I D X(B S)-1
\end{array}\right)\right. \\
& \left.+G_{1}\left(\begin{array}{c}
N-P H(P S 1) \\
I D X(B S)-1
\end{array}\right)+K_{1}\left(\begin{array}{c}
N-P H(P S 1) \\
I D X(B S)-2
\end{array}\right)\right\} \\
& -\left\{G_{1}\left(\begin{array}{c}
N-P H(P S 1) \\
I D X(B S)
\end{array}\right)+\left(K_{1}+1\right)\left(\begin{array}{c}
N-P H(P S 1) \\
I D X(B S)-1
\end{array}\right)\right\} \\
= & \left(G_{1}-1\right)\left(\begin{array}{c}
N-P H(P S 1) \\
I D X(B S)-1
\end{array}\right)+K_{1}\left(\begin{array}{c}
N-P H(P S 1) \\
I D X(B S)-2
\end{array}\right) \\
= & T_{1}\left(\begin{array}{c}
N-P H(P S 1) \\
I D X(B S)-1
\end{array}\right)+K_{1}\left(\begin{array}{c}
N-P H(P S 1) \\
I D X(B S)-2
\end{array}\right) \\
= & T_{1}\left(\begin{array}{c}
N-P H(P S)-1 \\
I D X(P T)
\end{array}\right)+K_{1}\left(\begin{array}{c}
N-P H(P S)-1 \\
I D X(P T)-1
\end{array}\right) \\
= & T_{1}\left(\begin{array}{c}
N-P H(P S)-P C H G(P S, P T) \\
I D X(P T)
\end{array}\right) \\
& +K_{1}\left(\begin{array}{c}
N-P H(P S)-P C H G(P S, P T) \\
I D X(P T)-1
\end{array}\right)
\end{aligned}
$$

The form $=\left(T_{1}+K_{1}\right) \rightarrow$ The formula holds.

3) If $M>1$ and $P T$ only has 1 change at $T_{M}$, then $P T=\left[1, G_{1}, \cdots, G_{M-1}, G_{M}-1\right]$

Let $\left.P S 1=\left[1, K_{1}, \cdots, K_{M-1}, K_{M}+1\right], 2.3\right) \rightarrow P H(P S 1)=P H(P S)+1$ :

$\operatorname{SUM}(N, P S, P T)=\operatorname{SUM}(N, P S)-\operatorname{SUM}(N, P S 1)$

$=\sum_{i=I D X(B S)-M}^{I D X(B S)} \sum A_{i}\left(\begin{array}{c}N-P H(P S) \\ i\end{array}\right)-\sum_{i=I D X(B S)-M}^{I D X(B S)} \sum B_{i}\left(\begin{array}{c}N-P H(P S 1) \\ i\end{array}\right)$

$=\sum_{i=I D X(B S)-M}^{I D X(B S)} \sum A_{i}\left\{\left(\begin{array}{c}N-P H(P S 1) \\ i\end{array}\right)+\left(\begin{array}{c}N-P H(P S 1) \\ i-1\end{array}\right)\right\}$

$-\sum_{i=I D X(B S)-M}^{I D X(B S)} \sum B_{i}\left(\begin{array}{c}N-P H(P S 1) \\ i\end{array}\right)$

$=\sum_{i=I D X(B S)-M}^{I D X(B S)}\left(\sum A_{i}-\sum B_{i}\right)\left(\begin{array}{c}N-P H(P S 1) \\ i\end{array}\right)+\sum A_{i}\left(\begin{array}{c}N-P H(P S 1) \\ i-1\end{array}\right)$

$=\sum_{i=I D X(B S)-M}^{I D X(B S)}\left(\sum A_{i}-\sum B_{i}\right)\left(\begin{array}{c}N-P H(P S)-1 \\ i\end{array}\right)+\sum A_{i}\left(\begin{array}{c}N-P H(P S)-1 \\ i-1\end{array}\right)$

$=\sum_{i=I D X(B S)-M-1}^{I D X(B S)} \sum C_{J}\left(\begin{array}{c}N-P H(P S)-1 \\ J\end{array}\right), J=i-1, \sum C_{J-1}=\sum A_{i+1}+\sum A_{i}-\sum B_{i}$

$=\sum_{J=I D X(P T)-M}^{I D X(P T)+1} \sum C_{J}\left(\begin{array}{c}N-P H(P S)-1 \\ J\end{array}\right)$

When $i=I D S(B S), \quad \sum C_{i}=\operatorname{MIN}(B S)-\operatorname{MIN}(B S)=0$

$=\sum_{J=I D X(P T)-M}^{I D X(P T)} \sum C_{J}\left(\begin{array}{c}N-P H(P S)-1 \\ J\end{array}\right)$

Use the symbol of (1.3)

$$
i=I D X(B S)-C N T, C N T=\text { Count of } X \in K
$$


Let $R(E)=\sum \prod_{L=1}^{M-1}\left(X_{L}+D_{L}\right), \quad E=$ Count of $\left\{X_{1}, \cdots, X_{M-1}\right\} \in K$

$\sum A_{i+1}=R(C N T-1)\left(G_{M}-(C N T-1)\right)$ $+R(C N T-2)\left(K_{M}+(M-1)-(C N T-2)\right)$

$\sum A_{i}=R(C N T)\left(G_{M}-C N T\right)+R(C N T-1)\left(K_{M}+(M-1)-(C N T-1)\right)$

$\sum B_{i}=R(C N T)\left(G_{M}-C N T\right)+R(C N T-1)\left(\left(K_{M}+1\right)+(M-1)-(C N T-1)\right)$

$\sum C_{J-1}=\sum A_{i+1}+\sum A_{i}-\sum B_{i}$

$=R(C N T-1)\left(G_{M}-C N T\right)+R(C N T-2)\left(K_{M}+M-C N T+1\right)$

$=R(C N T-1)\left(T_{M}-\{C N T-1\}\right)+R(C N T-2)\left(K_{M}+\{(M-1)-(C N T-2)\}\right)$

$\rightarrow$ Match of the form $\left(T_{1}+K_{1}\right)\left(T_{2}+K_{2}\right) \cdots\left(T_{M}+K_{M}\right)$

4) If $M>1$ and $P T$ only has 1 change at $T_{i<M}$, Let $R(E)=\sum \prod_{L=1, l \neq i}^{M}\left(X_{L}+D_{L}\right)$, use the same method of (3).

5) if $P C H G(P S, P T)>1$, Use 2.3) $\rightarrow$ divide the Items into subset $\rightarrow$ deducing by induction.

q.e.d.

Example:

$N-P H([1,3,5,7])-P C H G([1,3,5,7],[1,2,3,4])=N-(8-3-2)-3=N-6$

$\operatorname{SUM} \operatorname{SUBSET}(N,[1,3,5,7],[1,2,3,4])$

$\rightarrow$

form $=(2+3)(3+5)(4+7)$

$$
\begin{aligned}
& =24\left(\begin{array}{c}
N-6 \\
5
\end{array}\right)+108\left(\begin{array}{c}
N-6 \\
4
\end{array}\right)+174\left(\begin{array}{c}
N-6 \\
3
\end{array}\right)+105\left(\begin{array}{c}
N-6 \\
2
\end{array}\right) \\
& =1 \times 3 \times 5 \times 7+2 \times 4 \times 6 \times 8+3 \times 5 \times 7 \times 9+\cdots
\end{aligned}
$$

Among:

$24=2 \times 3 \times 4 ; 105=3 \times 5 \times 7$

$108=2 \times 3 \times(7+2)+2 \times(5+1) \times(4-1)+3 \times(3-1) \times(4-1)$

$174=2 \times(5+1) \times(7+1)+3 \times(3-1) \times(7+1)+3 \times 5 \times(4-2)$

Use the same method of 3.1)

3.2) Calculation formula of $S U M \_K(S E T(N, P S, P T), P F)$ is similar to 1.4).

Example:

SUM_SUBSET $(N,[1,3,7],[1,2,3])$

$=6\left(\begin{array}{c}N-6 \\ 4\end{array}\right)+2 \times(\{7\}+\{1\})\left(\begin{array}{c}N-6 \\ 3\end{array}\right)+3 \times(\{3-1\})\left(\begin{array}{c}N-6 \\ 3\end{array}\right)+3 \times 7\left(\begin{array}{c}N-6 \\ 2\end{array}\right)$

SUM_SUBSET $(10,[1,3,7],[1,2,3])$

$=1 \times 3 \times 7+2 \times 4 \times 8+3 \times 5 \times 9$

$=1 \times 3 \times 7+2 \times(3+1) \times(7+1)+3 \times(3+2) \times(7+2)$

$=\{1 \times 3 \times 7+2 \times 3 \times 7+3 \times 3 \times 7\}+\{2 \times 3 \times 1+3 \times 3 \times 2\}$

$+\{2 \times 1 \times 7+3 \times 2 \times 7\}+\{2 \times 1 \times 1+3 \times 2 \times 2\}$ 


$$
\begin{aligned}
& \{1 \times 3 \times 7+2 \times 3 \times 7+3 \times 3 \times 7\}=3 \times 7\left(\begin{array}{c}
10-6 \\
2
\end{array}\right) \\
& \{2 \times 3 \times 1+3 \times 3 \times 2\}=3 \times(\{3-1\})\left(\begin{array}{c}
N-6 \\
3
\end{array}\right)=6\left(\begin{array}{c}
10-6 \\
3
\end{array}\right) \\
& \{2 \times 1 \times 7+3 \times 2 \times 7\}=2 \times 7\left(\begin{array}{c}
N-6 \\
3
\end{array}\right)=14\left(\begin{array}{c}
N-6 \\
3
\end{array}\right) \\
& \{2 \times 1 \times 1+3 \times 2 \times 2\}=6\left(\begin{array}{c}
10-6 \\
4
\end{array}\right)+2 \times\{1\}\left(\begin{array}{c}
10-6 \\
3
\end{array}\right)
\end{aligned}
$$

3.3) Use the form $\left(T_{1}+K_{1}\right)\left(T_{2}+K_{2}\right) \cdots\left(T_{M}+K_{M}\right)=\sum X_{1} X_{2} \cdots X_{M}$ $S U M \_K\left(\operatorname{SET}(N, P S, P T), E_{1} E_{2} \cdots E_{M}\right)$$$
=\sum A_{q}\left(\begin{array}{c}
N-P H(P S)-P C H G(P S, P T) \\
I D X(P T)-q
\end{array}\right)
$$$$
A_{q}=\prod_{i=1}^{M} D_{i}, D_{i}=\left\{\begin{array}{l}
T_{i}-m: X_{i}=T_{i}, m=\text { count of }\left\{X_{2}, \cdots, X_{i-1}\right\} \in K \\
+m: X_{i}=K_{i}, m=\text { count of }\left\{X_{2}, \cdots, X_{i-1}\right\} \in T
\end{array}\right.
$$

$q=$ count of $X \in K, X_{1}=T_{1}, 2^{M-1}$ Items in total.

In particular:

If $P T 1=\left[1, T_{1}, \cdots, T_{M}\right]=[1,2, \cdots, M+1]$, then $P B(P S)=P C H G(P S, P T 1)$

$N-P H(P S)-P C H G(P S, P T 1)$

$=N-[I D X(P S)-2-P B(P S)]-P B(P S)$

$=N-\left(K_{M}-1\right)$

$I D X(P T 1)=M+2$

$\operatorname{SUM}\left(\operatorname{SET}(N, P S, P T 1), E_{1} \cdots E_{M}\right)$

$=2 \times 1^{M}+3 \times 2^{M}+\cdots+\left(N-K_{M}\right) \times\left(N-K_{M}-1\right)^{M}$

$\rightarrow$

$\operatorname{SUM}\left(\operatorname{SET}\left(N+K_{M}+1, P S, P T 1\right), E_{1} \cdots E_{M}\right)$

$=2 \times 1^{M}+3 \times 2^{M}+\cdots+(N+1) \times N^{M}$

3.4) $\sum_{n=1}^{N}(n+1) n^{M}=S U M_{-} K\left(\operatorname{SET}\left(N+K_{M}+1, P S, P T 1\right), E_{1} \cdots E_{M}\right)$

\section{Analysis of SUM_SUBSET(N,PS, [1, 2, ․, M + 1])}

$P S=\left[1, K_{1}, K_{2}, \cdots, K_{M}\right], P T 1=[1,2,3, \cdots, M+1]$. The simplest subset of $P S$ is $\operatorname{SET}(N, P S, P T 1)$.

$$
\begin{aligned}
& \text { SUM_SUBSET }(N, P S, P T 1) \\
& =\sum_{q=0}^{M} C_{q}\left(\begin{array}{c}
N-\left(K_{M}-1\right) \\
M+2-q
\end{array}\right)=\sum_{n=1}^{N-K_{M}} \sum_{q=0}^{M} C_{q}\left(\begin{array}{c}
n \\
M+1-q
\end{array}\right) \\
& =1 \times K_{1} \times \cdots \times K_{M}+2 \times\left(1+K_{1}\right) \times \cdots \times\left(1+K_{M}\right)+\cdots \\
& \quad+\left(N-K_{M}\right) \times\left(\left[N-K_{M}-1\right]+K_{1}\right) \times \cdots \times(N-1) \\
& =\sum_{n=1}^{N-K_{M}} n\left(n+K_{1}-1\right)\left(n+K_{2}-1\right) \cdots\left(n+K_{M}-1\right)
\end{aligned}
$$


Solve $\left(1^{\star}\right)$ in a normal way:

Decompose $n\left(n+K_{1}-1\right) \cdots\left(n+K_{M}-1\right)$ to $\sum_{j=1}^{M+1} D_{j}\left(\begin{array}{c}n \\ j\end{array}\right) \rightarrow D_{j}=C_{q}$, $q=M+1-j$

4.1) $\left.1<K_{1}<\cdots<K_{M}, 3.1\right)$ can decompose $n\left(n+K_{1}-1\right) \cdots\left(n+K_{M}-1\right)$ to $\sum_{j=1}^{M+1} D_{j}\left(\begin{array}{c}n \\ j\end{array}\right)$

In particular, 1.5) $\rightarrow C_{q}=(M+1) !\left(\begin{array}{c}M \\ M-q\end{array}\right) \rightarrow D_{j}=(M+1) !\left(\begin{array}{c}M \\ j-1\end{array}\right) \rightarrow$

4.2) $[x]^{M}=\frac{M !}{M !}\left(\begin{array}{c}M-1 \\ M-1\end{array}\right)[x]_{M}+\frac{M !}{(M-1) !}\left(\begin{array}{c}M-1 \\ M-2\end{array}\right)[x]_{M-1}+\cdots+\frac{M !}{1 !}\left(\begin{array}{c}M-1 \\ 0\end{array}\right)[x]_{1}$

4.3) $P$ is a prime number, $N-\left(K_{M}-1\right)=P$

1) $|\operatorname{SET}(N, P S, P T 1)|=P-1$,

2) $\begin{aligned} & \operatorname{SET}(N, P S, P T 1) \\ & =\left\{\left(1, K_{1}, \cdots, K_{M}\right),\left(2,1+K_{1}, \cdots, 1+K_{M}\right) \cdots(P-1, \cdots, N-1)\right\}\end{aligned}$

3) if $K_{M} \leq P-1$ and $P S \neq[1,2, \cdots, P-1]$, then

$S U M \_S U B S E T(N, P S, P T 1) \equiv 0 M O D P$

[Proof]

$$
\begin{aligned}
& |\operatorname{SET}(N, P S, P T 1)|=\left(\begin{array}{c}
N-P H(P S)-1-P C H G(P S, P T 1) \\
P B(P T 1)+1
\end{array}\right) \\
& =\left(\begin{array}{c}
\left(P+K_{M}-1\right)-\left(K_{M}+1-P B(P S)-2\right)-1-P C H G(P S, P T 1) \\
1
\end{array}\right) \\
& \stackrel{P B(P S)=P C H G(P S, P T 1)}{\longrightarrow} P-1 \rightarrow(1)(2)
\end{aligned}
$$

$\operatorname{SUM\_ SUBSET}(N, P S, P T 1)=\sum_{q=0}^{M} C_{q}\left(\begin{array}{c}P \\ I D X(P T 1)-q\end{array}\right)$

$K_{M} \leq P-1$ and $P S \neq[1,2, \cdots, P-1] \rightarrow I D X(P T 1)<P \rightarrow(3)$

q.e.d.

When $K_{M}=P-1$ and $P S \neq[1,2, \cdots, P-1]$

SUM_SUBSET $(N, P S, P T 1)$

$=1 \times K_{1} \times \cdots \times K_{M}+2 \times\left(1+K_{1}\right) \times \cdots \times P$

$+3 \times\left(2+K_{1}\right) \cdots\left(2+K_{M-1}\right)\left(2+K_{M}\right)$

$+4 \times\left(3+K_{1}\right) \cdots\left(3+K_{M-1}\right)\left(3+K_{M}\right)+\cdots$

$+(P-1) \times\left(P-2+K_{1}\right) \times \cdots \times\left(P-2+K_{M}\right)$

$3 \times\left(2+K_{1}\right) \cdots\left(2+K_{M-1}\right)\left(2+K_{M}\right) \equiv 1 \times 3 \times\left(2+K_{1}\right) \cdots\left(2+K_{M-1}\right)$

$4 \times\left(3+K_{1}\right) \cdots\left(3+K_{M-1}\right)\left(3+K_{M}\right)$

$\equiv 2 \times(1+[3]) \times\left(1+\left[2+K_{1}\right]\right) \cdots\left(1+\left[2+K_{M-1}\right]\right)$ 


$$
\begin{aligned}
& (P-1) \times\left(P-2+K_{1}\right) \cdots\left(P-2+K_{M}\right) \\
& \equiv(P-1) \times\left(P-2+K_{1}\right) \cdots\left(P-2+K_{M-1}\right)(2 P-3) \\
& \equiv(P-4+[1])(P-4+[3])\left(P-4+\left[2+K_{1}\right]\right) \cdots\left(P-4+\left[2+K_{M-1}\right]\right) \\
& 1 \times K_{1} \times \cdots \times K_{M} \equiv 1 \times K_{1} \times \cdots \times(P-1) \equiv(P+1)\left(P+K_{1}\right) \cdots(P+P-1) \\
& \equiv(P-1)(P-2+3)\left([P-2]+\left[2+K_{1}\right]\right) \cdots\left([P-2]+\left[2+K_{M-1}\right]\right) \\
& \rightarrow
\end{aligned}
$$

$S U M \_S U B S E T(N, P S, P T 1)$

$\equiv S U M \_S U B S E T\left(N,\left[1,3,2+K_{1}, \epsilon, 2+K_{M-1}\right], P T 1\right) M O D P$

$P S=\left[1, K_{1} \cdots K_{M}\right]$ can be slided to $\left[1,3,2+K_{1}, \cdots, 2+K_{M-1}\right]$ by $M O D P$

If $K_{M}=P-1$ and exists $K_{i+1}-K_{i}>2, P S$ can be slided to $[1, \cdots, X<P-1]$

If $K_{M}=P-1$ and not exists $K_{i+1}-K_{i}>2, P S$ must be a Basic Shape, can only be slided to $[1, \cdots, X=P-1]$

\section{Define:}

A Basic Shape $P S=\left[1, K_{1} \cdots K_{M}\right]=\llbracket L_{1} L_{2} \cdots L_{Q} \rrbracket$, among:

$L_{i}=$ count of continuity. $\left(L_{i}, L_{i+1}\right)$ means a discontinuity, there are $Q-1$ discontinuities.

Example: $[1,3,5]=\llbracket 1,1,1 \rrbracket,[1,2,3,5]=\llbracket 3,1 \rrbracket,[1,2,4,5]=\llbracket 2,2 \rrbracket$

Obvious: $\llbracket L_{1} L_{2} \cdots L_{Q} \rrbracket$ can been slided to $\left[L_{Q}, L_{1}, L_{2}, \cdots\right]$, $\left[L_{Q-1}, L_{Q}, L_{1}, L_{2}, \cdots\right], \cdots$

4.4) $P S$ is a Basic Shape, IDX $(P S)=P, P S \neq[1,2, \cdots, P-1],\{P S 1, P S 2, \cdots\}$ are all shapes that $P S$ can scroll to, then $\operatorname{MIN}(P S 1)+\operatorname{MIN}(P S 2)+\cdots \equiv 0 M O D P$. This is a promotion of 1.6)

[Proof]

$$
\begin{aligned}
& \quad 3 \times\left(2+K_{1}\right) \cdots\left(2+K_{M-1}\right)\left(2+K_{M}\right) \equiv 1 \times 3 \times\left(2+K_{1}\right) \cdots\left(2+K_{M-1}\right) M O D P \\
& \text { If } 2+K_{M-1} \neq P,\left[1,3,\left(2+K_{1}\right), \cdots,\left(2+K_{M-1}\right)\right] \in\{P S 1, P S 2, \cdots\} \\
& \cdots \\
& \text { SUM_SUBSET }(N, P S 1, P T 1) \equiv \operatorname{MIN}(P S 1)+\operatorname{MIN}(P S 2)+\cdots \equiv 0 M O D P
\end{aligned}
$$

q.e.d.

Example:

$$
\begin{aligned}
& 1 \times(3 \times 4 \times 5) \times(7 \times 8) \times 10+1 \times 3 \times(5 \times 6 \times 7) \times(9 \times 10) \\
& +(1 \times 2) \times 4 \times 6 \times(8 \times 9 \times 10)+(1 \times 2 \times 3) \times(5 \times 6) \times 8 \times 10 \\
& =139260 \equiv 0 \text { MOD } 11
\end{aligned}
$$

\section{Calculation Formula of $1^{M}+2^{M}+3^{M}+\cdots+N^{M}$}

Use the form $\left(T_{1}+K_{1}\right)\left(T_{2}+K_{2}\right) \cdots\left(T_{M}+K_{M}\right)$.

In general, $K_{i}$ and $K_{j}$ cannot be exchanged, but when $P T=P T 1=[1,2, \cdots, M+1]$,

$$
S U M \_S U B S E T(N, P S, P T 1)=\sum_{q=0}^{M} C_{q}\left(\begin{array}{c}
N-P H(P S)-P C H G(P S, P T 1) \\
I D X(P T 1)-q
\end{array}\right)
$$




$$
C_{q}=\sum \prod_{i=1}^{M}\left(X_{i}+D_{i}\right)=\sum \prod_{\text {choice } M-q \text { from } T}(T-D) \prod_{\text {choice } q \text { from } K}(K+D)
$$

Easy to see: $\prod_{\text {choice } M-q \text { from } T}(T-D)=(M+1-q)$ !

Can prove: $\left(K_{1}, K_{2}, \cdots, K_{M}\right)$ is permutable in $\sum \prod_{\text {choice } q \text { from } K}(K+D)$

$\rightarrow\left(K_{1}, K_{2}, \cdots, K_{M}\right)$ is permutable in the form.

SUM_SUBSET $(N, P S, P T 1)$

$$
\begin{aligned}
& =\sum_{q=0}^{M} A_{q}\left(\begin{array}{c}
N-K_{M}+1 \\
M+2-q
\end{array}\right)=\sum_{n=K_{M}+1}^{N} \sum_{q=0}^{M} A_{q}\left(\begin{array}{c}
n-K_{M} \\
M+1-q
\end{array}\right) \\
& =1 \times K_{1} \times \cdots \times K_{M}+2 \times\left(1+K_{1}\right) \times \cdots \times\left(2+K_{M}\right)+\cdots
\end{aligned}
$$

Add one more factor $K_{i}$ to the end:

$$
\begin{aligned}
1 \times K_{1} \times \cdots \times K_{M} \times K_{i}+2 \times\left(1+K_{1}\right) \times \cdots \times\left(1+K_{M}\right) \times\left(1+K_{i}\right)+\cdots & \\
= & \sum_{n=K_{M}+1}^{N} \sum_{q=0}^{M} A_{q}\left(\begin{array}{c}
n-K_{M} \\
M+1-q
\end{array}\right)\left(n-1-K_{M}+K_{i}\right) \\
= & \sum_{n=K_{M}+1}^{N} \sum_{q=0}^{M} A_{q}\left(\begin{array}{c}
n-K_{M} \\
M+1-q
\end{array}\right)\left(\left[n-K_{M}+1\right]+\left[K_{i}-2\right]\right) \\
= & \sum_{n=K_{M}+1}^{N} \sum_{q=0}^{M}\left\{A_{q}(M+2-q)\left(\begin{array}{c}
n-K_{M}+1 \\
M+2-q
\end{array}\right)+A_{q}\left(K_{i}-2\right)\left(\begin{array}{c}
n-K_{M} \\
M+1-q
\end{array}\right)\right\} \\
= & \sum_{q=0}^{M} A_{q}(M+2-q)\left(\begin{array}{c}
N-K_{M}+2 \\
M+3-q
\end{array}\right)+\sum_{q=0}^{M} A_{q}\left(K_{i}-2\right)\left(\begin{array}{c}
N-K_{M}+1 \\
M+2-q
\end{array}\right) \\
= & \sum_{q=0}^{M} A_{q}(M+2-q)\left(\begin{array}{c}
N-K_{M}+1 \\
M+3-q
\end{array}\right) \\
& +\sum_{q=0}^{M}\left\{A_{q}(M+2-q)+A_{q}\left(K_{i}-2\right)\right\}\left(\begin{array}{c}
N-K_{M}+1 \\
M+2-q
\end{array}\right) \\
= & \sum_{q=0}^{M} A_{q}(M+2-q)\left(\begin{array}{c}
N-K_{M}+1 \\
M+3-q
\end{array}\right) \\
& +\sum_{q=0}^{M} A_{q}\left(K_{i}+(M-q)\right)\left(\begin{array}{c}
N-K_{M}+1 \\
M+3-(q+1)
\end{array}\right)
\end{aligned}
$$

Let $P S 2=\left[1, K_{1}, \cdots, K_{M}, K_{M+1}=K_{i}\right]$,

$P T 2=\left[1, T_{1}, \cdots, T_{M}, T_{M+1}\right]=[1,2, \cdots, M+1, M+2]$

$I D X(P T 2)=M+3, N-P H(P S 2)-P C H G(P S 2, P T 2)=N-K_{M}+1$

$q=$ count of $\left\{X_{1}, \cdots, X_{M}\right\} \in K$

$A_{q}(M+2-q)=A_{q}\left(T_{M+1}-q\right)$ means $X_{M+1}=T_{M+1}, A_{q}\left(K_{i}+(M-q)\right)$

means $X_{M+1}=K_{M+1}$

It's match the form $\left(T_{1}+K_{1}\right)\left(T_{2}+K_{2}\right) \cdots\left(T_{M}+K_{M}\right)\left(T_{M+1}+K_{i}\right)$

Recursion $\rightarrow$

5.1) $K_{i}<K_{j}, K_{i}=K_{j}, K_{i}>K_{j}$ are allowed,

$\operatorname{SUM} \operatorname{SUBSET}(N, P S, P T 1)$ can use the form $\left(T_{1}+K_{1}\right)\left(T_{2}+K_{2}\right) \cdots\left(T_{M}+K_{M}\right)$

Example:

The form $=(2+3)(3+5)(4+3) \rightarrow$

SUM_SUBSET $(N,[1,3,5,3],[1,2,3,4])$

$=24\left(\begin{array}{c}N-4 \\ 5\end{array}\right)+84\left(\begin{array}{c}N-4 \\ 4\end{array}\right)+102\left(\begin{array}{c}N-4 \\ 3\end{array}\right)+45\left(\begin{array}{c}N-4 \\ 2\end{array}\right)$ 
Among:

$45=3 \times 5 \times 3$

$102=3 \times 5 \times(4-2)+3 \times(3-1) \times(3+1)+2 \times(5+1) \times(3+1)$

$84=2 \times 3 \times(3+2)+2 \times(5+1) \times(4-1)+3 \times(3-1) \times(4-1)$

SUM_SUBSET $(9,[1,3,5,3],[1,2,3,4])$

$=1 \times 3 \times 3 \times 5+2 \times 4 \times 4 \times 6+3 \times 5 \times 5 \times 7+4 \times 6 \times 6 \times 8=1914$

$=24\left(\begin{array}{l}5 \\ 5\end{array}\right)+84\left(\begin{array}{l}5 \\ 4\end{array}\right)+102\left(\begin{array}{l}5 \\ 3\end{array}\right)+45\left(\begin{array}{l}5 \\ 2\end{array}\right)$

The form $=(2+4)(3+7)(4+7) \rightarrow$

SUM_SUBSET $(N,[1,4,7,7],[1,2,3,4])$

$=24\left(\begin{array}{c}N-6 \\ 5\end{array}\right)+126\left(\begin{array}{c}N-6 \\ 4\end{array}\right)+248\left(\begin{array}{c}N-6 \\ 3\end{array}\right)+196\left(\begin{array}{c}N-6 \\ 2\end{array}\right)$

Among:

$196=4 \times 7 \times 7$

$248=4 \times 7 \times(4-2)+4 \times(3-1) \times(7+1)+2 \times(7+1) \times(7+1)$

$126=2 \times 3 \times(7+2)+2 \times(7+1) \times(4-1)+4 \times(3-1) \times(4-1)$

SUM_SUBSET $(12,[1,4,7,7],[1,2,3,4])$

$=1 \times 4 \times 7 \times 7+2 \times 5 \times 8 \times 8+3 \times 6 \times 9 \times 9+4 \times 7 \times 10 \times 10+5 \times 8 \times 11 \times 11$

$=9934=24\left(\begin{array}{l}6 \\ 5\end{array}\right)+126\left(\begin{array}{l}6 \\ 4\end{array}\right)+248\left(\begin{array}{l}6 \\ 3\end{array}\right)+196\left(\begin{array}{l}6 \\ 2\end{array}\right)$

In particular:

5.2) $\sum_{n=1}^{M} n^{M}=S U M \_S U B S E T(N+1,[1,1, \cdots, 1],[1,2, \cdots, M])$

Example:

$$
(N+1)-P H(P S)-P C H G(P S, P T 1)=(N+1)-0-0=N+1
$$

The form $=(2+1)$

$\rightarrow 1^{2}+2^{2}+\cdots+N^{2}=2\left(\begin{array}{c}N+1 \\ 3\end{array}\right)+\left(\begin{array}{c}N+1 \\ 2\end{array}\right)=\frac{N(N+1)(2 N+1)}{6}$

The form $=(2+1)(3+1)$

$\rightarrow 1^{3}+2^{3}+\cdots+N^{3}=6\left(\begin{array}{c}N+1 \\ 4\end{array}\right)+6\left(\begin{array}{c}N+1 \\ 3\end{array}\right)+\left(\begin{array}{c}N+1 \\ 2\end{array}\right)=\frac{N^{2}(N+1)^{2}}{4}$

The form $=(2+1)(3+1)(4+1)$

$\rightarrow 1^{4}+2^{4}+\cdots+N^{4}=24\left(\begin{array}{c}N+1 \\ 5\end{array}\right)+36\left(\begin{array}{c}N+1 \\ 4\end{array}\right)+14\left(\begin{array}{c}N+1 \\ 3\end{array}\right)+\left(\begin{array}{c}N+1 \\ 2\end{array}\right)$

Among:

$$
\begin{aligned}
& 14=2 \times(1+1) \times(1+1)+1 \times 1 \times(4-2)+1 \times(3-1) \times(1+1) \\
& 36=2 \times 3 \times(1+2)+2 \times(1+1) \times(4-1)+1 \times(3-1) \times(4-1)
\end{aligned}
$$

$S(M, K)$ is Stirling number of the second kind, 
Definition of $S(M, K) \rightarrow \sum_{n=1}^{N} n^{M}=\sum_{K=1}^{M} K ! S(M, K)\left(\begin{array}{l}N+1 \\ K+1\end{array}\right)$

It's equal to 5.2), so we have a way to calculate $S(M, K)$.

3.4) can be seen as

$\sum_{n=1}^{N}(n+1) n^{M}=\operatorname{SUM} \operatorname{SUBSET}(N+1,[1,0, \cdots, 0],[1,2, \cdots, M+1])$

\section{Conflicts of Interest}

The author declares no conflicts of interest regarding the publication of this paper.

\section{References}

[1] Peng, J. (2020) Shape of Numbers and Calculation Formula of Stirling Numbers. Open Access Library Journal, 7, 1-11. https://doi.org/10.4236/oalib.1106081

[2] Peng, J. (2020) Subdivide the Shape of Numbers and a Theorem of Ring. Open Access Library Journal, 7, 1-14. https://doi.org/10.4236/oalib.1106719 